\title{
Die navigierte Reposition und Marknagelosteosynthese von Femurschaftfrakturen
}

\author{
T. Hüfner, J. Geerling, D. Kendoff, T. Gösling, C. Krettek
}

\section{Zusammenfassung}

Die geschlossene Reposition und Marknagelosteosynthese von Femurschaftfrakturen ist ein etabliertes und minimal invasives Verfahren. Jedoch erfolgen die Repositionskontrolle, die Nagelinsertion und die Verriegelung alleine fluoroskopiegestützt und belasten damit v. a. das OP-Personal. Weiterhin ist die intraoperative Rotationskontrolle noch nicht befriedigend gelöst.

Die fluoroskopiegestützte Navigation ermöglicht den quantitativen Ver- gleich der Rotation mit der nichtbetroffenen kontralateralen unteren Extremität. Für die Reposition wird zunächst eine Referenzbasis am proximalen und am distalen Fragment montiert. Die notwendigen Aufnahmen werden im dislozierten Zustand mit dem navigierten Fluoroskop durchgeführt und das Fluoroskop anschließend zurückgefahren. Der Femurmarknagel wird ebenfalls mit der navigierten Halterung eingebracht. Nach der distalen Verriegelung erfolgen die Rotationskontrolle und die konventionelle Beendigung der Operation.

\section{Einleitung}

Die geschlossene Reposition und interne Fixation von Femurschaftfrakturen ist ein Standardverfahren in der Traumatologie. Die geringe Invasivität geht mit einer erhöhten intraoperativen Strahlenbelastung für das Personal und den Patienten einher [1, 8, 9, 15, 17]. Achsabweichungen in der a.p- und seitlichen Ebene können gut kontrolliert werden, jedoch stellt die korrekte Einstellung der Rotation ein weiteres Problem dar [2, 5, 7, 10, 17]. Erst in der postoperativen CT-Untersuchung kann die Rotation als Anteversion des Schenkelhalses relativ zur Condylenebene distal präzise gemessen werden [4, $6,11,12,14]$. Rotationsfehler, gemessen als Differenz zur Gegenseite über $15^{\circ}$ führen zu Beschwerden, Bewegungseinschränkungen und langfristig zu einer Coxarthrose. Für eine Korrektur eines entsprechenden Rotationsfehlers ist ein erneuter Eingriff erforderlich.

OP-JOURNAL 2002; 17: 36-38

(c) Georg Thieme Verlag Stuttgart · New York
Mit der Navigation von Femurschaftfrakturen können einerseits die Reposition, andererseits die Implantation des Femurmarknagels und die distale Verriegelung präzise durchgeführt werden. Dabei kann bereits intraoperativ die Rotation mit der Gegenseite quantitativ verglichen werden [4].

Darüber hinaus wird beispielsweise bei der Verriegelung die intraoperative Strahlenbelastung um bis zu 90\% vermindert [13].

Die Röntgenaufnahmen werden mit dem navigierten Fluoroskop vor der eigentlichen Operation aufgenommen und in das Navigationssystem importiert und dort auf dem Monitor dargestellt. Die Instrumente werden in Echtzeit im Bild des Patienten dargestellt. Die simultane Darstellung in mehreren Ansichten ermöglicht die genaue Platzierung von Implantaten. Dies geschieht ohne Röntgenstrahlung, da die initialen Bilder verwendet werden, d.h. die totale Röntgenstrahlenbelastung ist signifikant kleiner, verglichen mit einer konventionellen Operation ohne Navigation. Da das Fluoroskop nicht mehr benötigt wird, kann er vom Operationstisch entfernt werden und stellt somit keine Behinderung mehr dar. Dank einer „virtuellen Tastatur“ kann der Chirurg steril vom Operationstisch aus das System steuern.

\section{Präoperative Vorbereitung}

Die Indikation stellen Femurschaft-, Quer- und Schrägfrakturen mit insgesamt 2 Fragmenten dar. Ein drittes Fragment kann zur Zeit nicht navigiert werden. Die Patienten sollten in einem stabilen Zustand sein, da die navigierte Femurmarknagel-Osteosynthese einen zusätzlichen Zeitaufwand bedeutet.

Indikation zur navigierten Femurmarknagel-Osteosynthese: Femurschaftfrakturen mit insgesamt Fragmenten.

Die Instrumente unterscheiden sich grundsätzlich nicht von den konventionellen, sind aber mit je einer Referenzbasis versehen (Abb. 1).

Die Lagerung unterscheidet sich ebenfalls nicht von der konventionellen Operationstechnik, der Patient wird auf dem Normaltisch in Rückenlage gelagert. Zunächst wird in Rückenlage das nicht betroffene Bein mit dem navigierten Fluoroskop geröntgt: das proximale und das di-

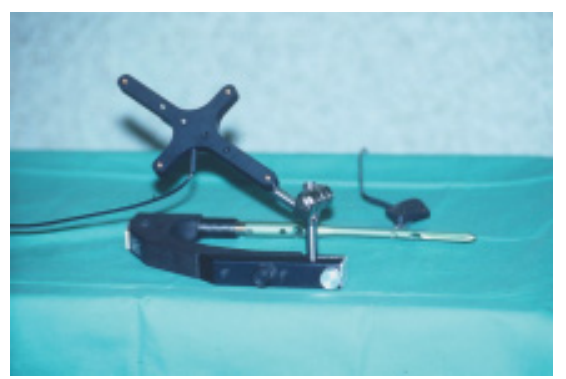

Abb.1 Navigierte Instrumente für die Femurnagelosteosynthese (UFN), Fa. Synthes, Bochum). Navigationssystem Surgigate ${ }^{\circledR}, \mathrm{Fa}$. Medivision, Umkirch. 
stale Femur in 2 Ebenen, die Bilder werden in das Navigationssystem importiert und die Antetorsion (zum postoperativen Rotationsvergleich) gemessen. Dazu wird die Referenzbasis hilfsweise an den OPTisch montiert und während der Bildakquisition das Bein gehalten. Anschließend wird das nicht betroffene Bein in einer „gynäkologischen“ Stütze abgewinkelt ausgelagert. Vor der Abdeckung muss überprüft werden, ob mit dem Fluoroskop auf der verletzten Seite das proximale Femur, die Frakturzone und das distale Femur in beiden Ebenen geröntgt werden können.

Die Instrumente und die Lagerung unterscheiden sich grundsätzlich nicht von der konventionellen Operation, es ist aber mit einem höheren Zeitaufwand bei der Operation zu rechnen.

\section{Navigation}

In einem ersten Schritt werden über Stichinzisionen die dynamischen Referenzbasen am proximalen und distalen Fragment montiert. Es ist darauf zu achten, dass eine absolute Stabilität besteht, gegebenenfalls muss die Montage durch eine zweite Schanz-Schraube verstärkt werden (Abb. 2). Es werden wie auf der gesunden Seite das proximale und das distale Femur und zusätzlich die Frakturzone in beiden Ebenen abgebildet (Abb. 3). Dabei müssen die Referenzbasen, das Lot und die Referenzbasis des Fluoroskops simultan für die OptotrakKamera sichtbar sein. Das Fluoroskop kann jetzt zurückgefahren werden, da zunächst zusätzliche Aufnahmen für die $\mathrm{Na}$ -

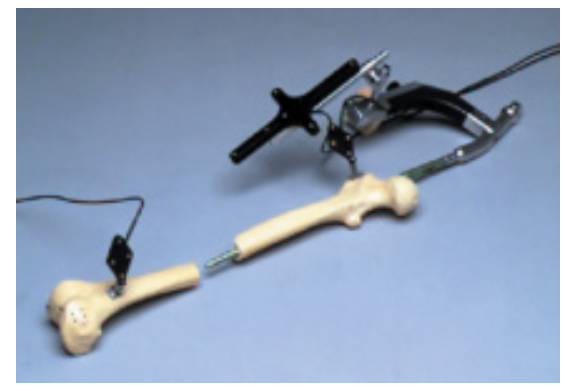

Abb. 2 Montage der Referenzbasen an das proximale und distale Fragment (Modell).

vigation nicht mehr erforderlich sind. Nach Montage des Femurmarknagels an die navigierte Halterung erfolgt die Kalibrierung (Längenabgleich). Das System misst nun die Position der Instrumente relativ zur Referenzbasis. Über die konventionelle Inzision für die Nagelinsertion wird der navigierte Nagel eingebracht. Dabei muss auf eine direkte Sichtverbindung der Referenzbasen des Marknagels und der Fragmente zur Kamera geachtet werden.

Die Reposition erfolgt unter Monitorkontrolle des Navigationssystems, d.h. die Fragmentachsen müssen deckungsgleich gebracht werden und anschließend der abgebildete Nagel vorangetrieben werden (Abb. 4). Nach Insertion in das distale Fragment erfolgt eine konventionelle Kontrolle mit dem Fluoroskop, um die virtuelle Abbildung mit der Realität abzugleichen (Verbiegung). Der Nagel wird schließlich in die endgültige Position vorgeschlagen.
Nach initialen Röntgenaufnahmen ist für die Reposition und Marknagelosteosynthese keine weitere Aufnahme notwendig, empfohlen werden aber gelegentliche Aufnahmen mit dem Fluoroskop zur Kontrolle der Navigation.

\section{Verriegelung}

Für die Verriegelung werden zwei navigierte Aufnahmen (a.p. und seitlich) des distalen Nagelendes im Femur aufgenommen und das Fluoroskop wieder zurückgefahren.

Die navigierte Bohrmaschine wird nach Montage des Bohrers kalibriert (Längenabgleich) und unter direkter Sichtverbindung der Bohrmaschine und der Referenzbasis des distalen Fragmentes zur Kamera erfolgt die Bohrung für die distale Verriegelung (Abb. 5).

\section{Rotationskontrolle}

Vor der proximalen Verriegelung erfolgt die Rotationskontrolle mit dem Navigationssystem. Es werden erneut vier navigierte Aufnahmen durchgeführt (proximales und distales Femur in 2 Ebenen). Nachdem eine seitengleiche Rotation eingestellt wurde, wird die Operation konventionell beendet mit ggf. Zurückschlagen des Nagels und proximaler Verriegelung.

Ein entscheidender Vorteil der Navigation ist die erstmalig mögliche intraoperative quantitative Rotationskontrolle.

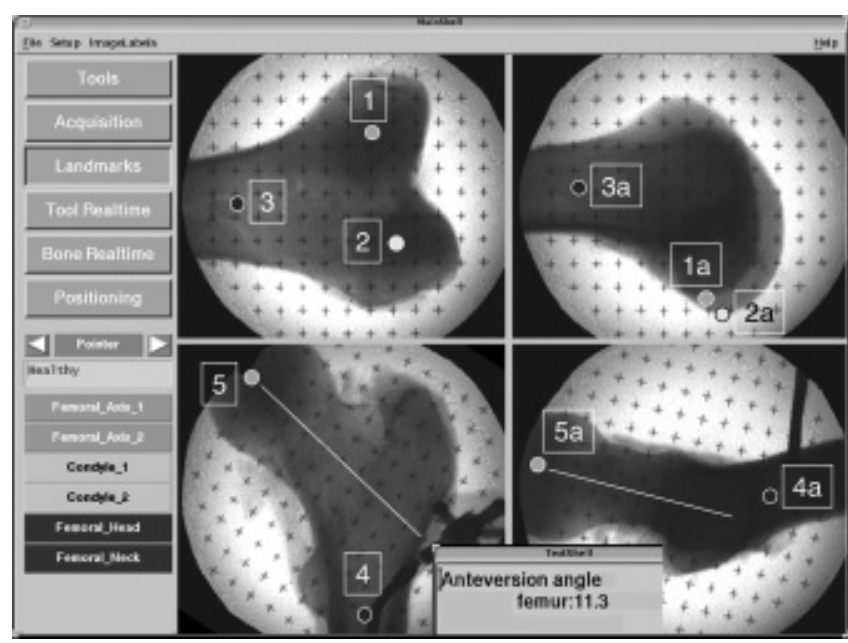

Abb. 3 Bildakquisition vom proximalen und distalen Femur in beiden Ebenen. Der Antetorsionswinkel wird für die gesunde Seite vor der Operation und für die verletzte Seite nach der distalen Verriegelung bestimmt.

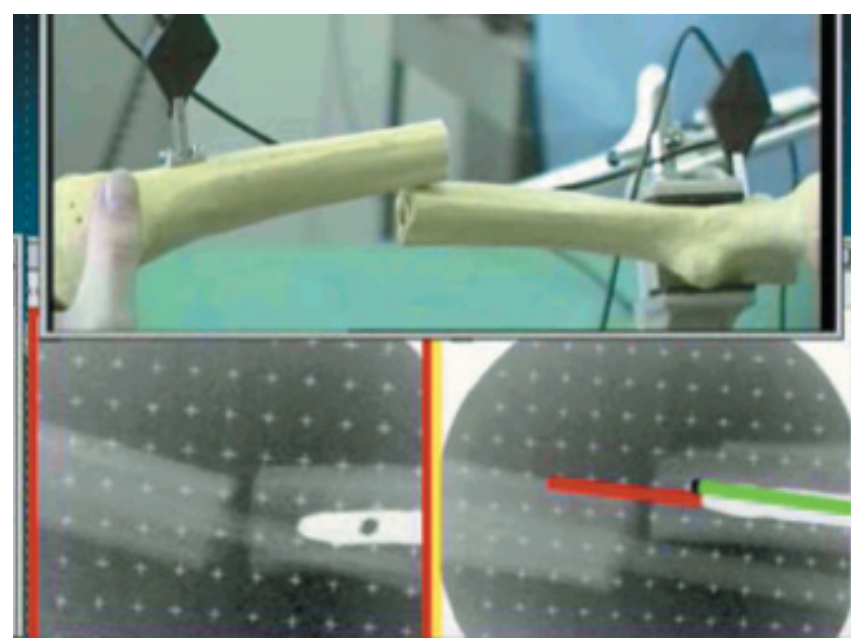

Abb. 4 Bei der Reposition werden die Fragmentachsen grün und der Nagel als rote Achse dargestellt. Eine Dynamisierung der Röntgenbilder der Fragmente selbst ist bisher im Laborversuch realisiert worden, aber noch nicht kommerziell erhältlich. 


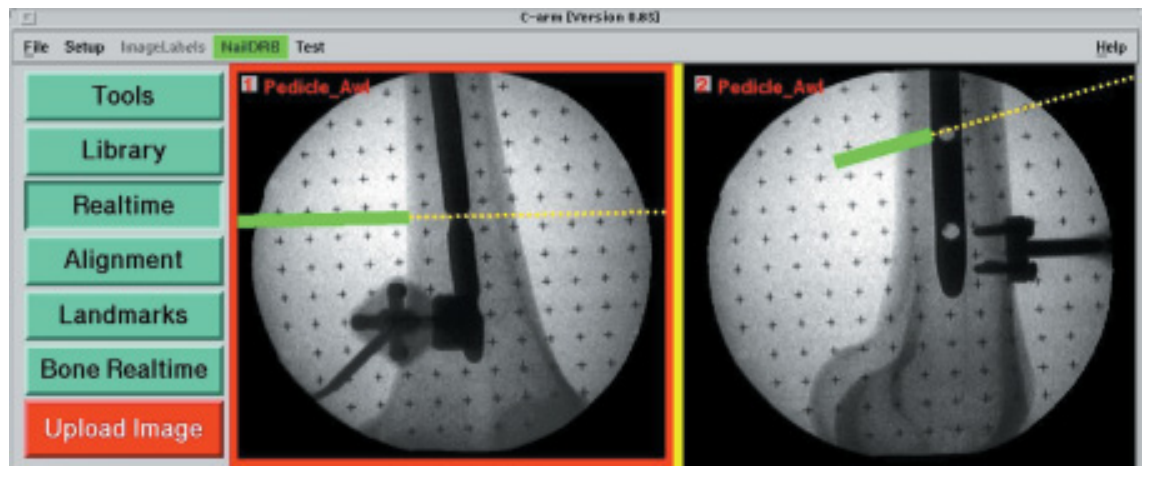

Abb.5 Distale Verriegelung. Die Röntgenbilder werden vor der Verriegelung aufgenommen und importiert. Für die Bohrung selbst (Bohrer ist als grüne Achse dargestellt) ist keine weitere Bildgebung erforderlich.

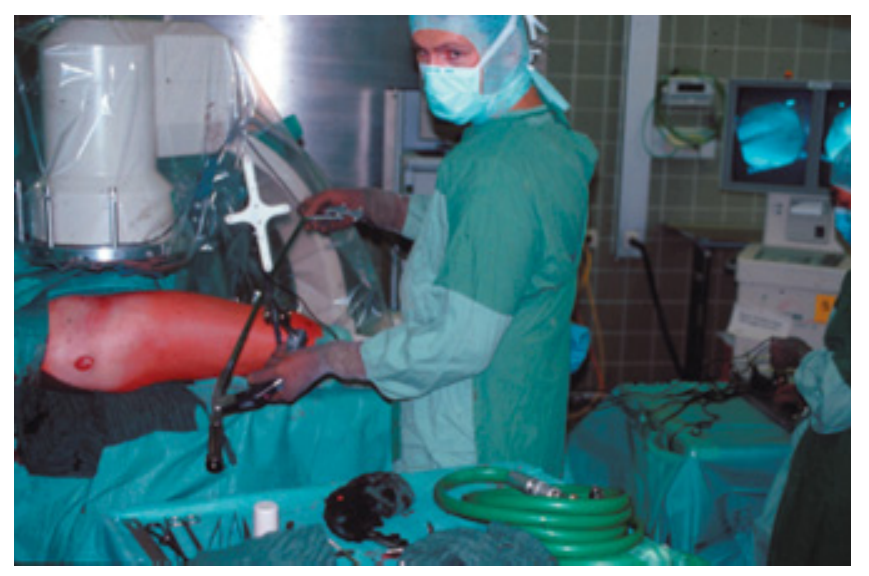

\section{Diskussion}

Die navigierte Reposition und Marknagelosteosynthese bei Femurschaftfrakturen ist ein neues Konzept, welches kommerziell Ende 2001 eingeführt wurde. Grundsätzliche Probleme, insbesondere intraoperative Strahlenbelastung und Rotationskontrolle bei der konventionellen Operationstechnik können mit der Navigation entscheidend verbessert werden.

Demgegenüber stehen Probleme bei dem navigierten Verfahren. Es bedarf einer exakten Planung der Ergonomie im Operationssaal, denn die direkten Sichtverbindungen der Referenzbasen am Patientenknochen und die der Instrumente zur Kamera sind essenziell. Ein weiteres Problem stellt die manchmal ungenügende Bildqualität der importierten Bilder vom Fluoroskop dar. Gelegentlich sollte eine konventionelle Überprüfung der aktuellen Situation auf dem Bildschirm des Navigationssystems mit dem Fluoroskop erfolgen. Gewöhnungsbedürftig ist die Darstellung der Fragmente als reine Achsen und des Marknagels als singuläre andersfarbige Achse.
Abb. 6 Vor der Nagelinsertion muss eine Kalibrierung der Länge des Nagels erfolgen. Dazu müssen die Referenzbasen der Instrumente Sichtkontakt zur optoelektronischen Kamera haben.

${ }^{5}$ Jäger M, Schmidt JM (1982h) Rotationsfehlstellung nach Oberschenkelmarknagelung. Hefte Unfallheilkd 158: 198-

${ }^{6}$ Jend HH (1986l) Die computertomographische Antetorsionswinkelbestimmung. Fortschr. Röntgenstr. 144: 447-452

${ }^{7}$ Krettek C, Rudolf J, Schandelmaier P, Guy P, Könemann B, Tscherne H (1996g) Unreamed intramedullary nailing of femoral shaft fractures: operative technique and early clinical experience with the standard locking option. Injury 27: 233-254

${ }^{8}$ Krettek C, Schandelmaier P, Miclau T, Bertram R, Holmes W, Tscherne H (1997c) Transarticular joint reconstruction and indirect plate osteosynthesis for complex distal supracondylar femoral fractures. Injury 28 (Suppl. 1): 31-41

${ }^{9}$ Krettek C, Schandelmaier P, Miclau T, Tscherne H (1997b) Minimally invasive percutaneous plate osteosynthesis (MIPPO) using the DCS in proximal and distal femoral fractures. Injury 28 (Suppl. 1): 20-30

${ }^{10}$ Laer L (1977i) Beinlängendifferenzen und Rotationsfehler nach Oberschenkelschaftfrakturen im Kindesalter. Arch.Orthop.Unfallchir. 89:121-137

${ }^{11}$ Mesgarzadeh M, Revesz G, Bonakdarpour A (1987m) Femoral neck torsion angle measurement by computed tomography. J.Comput.Assist.Tomogr. 11: 799-803

${ }^{12}$ Strecker W, Franzreb M, Pfeiffer T, Pokar S, Wikström M, Kinzl L (1994n) Computertomographische Torsionswinkelbestimmung der unteren Extremitäten. Unfallchirurg 97: 609-613

13. Suhm N, Jacob AL, Zuna I, Roser HW, Regazzoni P, Messmer P, [Radiation exposure of the patient by intraoperative imaging of intramedullary osteosyntheses]. Radiologe, 2001. 41(1): p. $91-4$.

${ }^{14}$ Waidelich HA, Strecker W, Schneider E (1992o) Computertomographische Torsionswinkel- und Langenmessung an der unteren Extremitat. Methodik, Normalwerte und Strahlenbelastung. Rofo Fortschr.Geb.Rontgenstr.Neuen Bildgeb.Verfahr. 157: 245-251

${ }^{15}$ Wenda K, Degreif J, Runkel M, Ritter G (1994d) Zur Technik der Plattenosteosynthese des Femurs. Unfallchirurg 97: 13-18

Die korrekte Anwendung erfordert eine Schulung des ärztlichen und pflegerischen Personals. Dann sind eine optimale Nutzung und sehr gute Ergebnisse zu erwarten.

\section{Literaturverzeichnis}

${ }^{1}$ Bone LB, Anders MJ, Rohrbacher BJ (1998a) Treatment of femoral fractures in the multiply injured patient with thoracic injury. Clinical Orthopaedics and Related Research: 57-61

2 Braten M, Terjesen T, Rossvoll I (1993f) Torsional deformity after intramedullary nailing of femoral shaft fractures. Journal of Bone and Joint Surgery [Br] 75: 799-803

${ }^{3}$ Hernandez RJ, Tachdjian MO, Poznanski AK, Dias LS (1981k) CT determination of femoral torsion. AJR Am. J. Roentgenol. 137: 97-101

${ }^{4}$ Hofstetter R, Slomczykowski M, Krettek C, Koppen G, Sati M, Nolte LP, Computer-assisted fluoroscopy-based reduction of femoral fractures and antetorsion correction. Comput Aided Surg, 2000.5 (5): p. $311-25$. "durchgeschobene“ ' Kondylenplatte. Unfallchirurgie 21: 77-82

${ }^{17}$ Wolf H, Schauwecker F, Tittel K (1984j) Rotationsfehler nach Marknagelung des Oberschenkels. (Malrotation following intramedullary nailing of the femur). Unfallchirurgie 10: $133-136$

\section{Dr. Tobias Hüifner}

Assistenzarzt

Jens Geerling

AIP

\section{Daniel Kendoff}

AIP

\section{Thomas Gösling}

Assistenzarzt

Christian Krettek

Direktor der Klinik

Unfallchirurgische Klinik

Medizinische Hochschule Hannover

Carl-Neuberg-Str. 1

D-30625 Hannover 